\title{
A!
}

This is an electronic reprint of the original article.

This reprint may differ from the original in pagination and typographic detail.

Ofodile, Ikechukwu; Ehrpais, Hendrik; Slavinskis, Andris; Anbarjafari, Gholamreza

\section{Stabilised LQR control and optimised spin rate control for nanosatellites}

\section{Published in:}

Proceedings of 9th International Conference on Recent Advances in Space Technologies, RAST 2019

DOI:

10.1109/RAST.2019.8767850

Published: 01/06/2019

Document Version

Peer reviewed version

Please cite the original version:

Ofodile, I., Ehrpais, H., Slavinskis, A., \& Anbarjafari, G. (2019). Stabilised LQR control and optimised spin rate control for nanosatellites. In S. Menekay, O. Cetin, \& O. Alparslan (Eds.), Proceedings of 9th International Conference on Recent Advances in Space Technologies, RAST 2019 (pp. 715-722). [8767850] IEEE. https://doi.org/10.1109/RAST.2019.8767850

This material is protected by copyright and other intellectual property rights, and duplication or sale of all or part of any of the repository collections is not permitted, except that material may be duplicated by you for your research use or educational purposes in electronic or print form. You must obtain permission for any other use. Electronic or print copies may not be offered, whether for sale or otherwise to anyone who is not an authorised user. 


\section{Stabilised LQR Control and Optimised Spin Rate Control for Nanosatellites}

\author{
Ikechukwu Ofodile \\ Tartu Observatory and iCV Lab \\ University of Tartu, Estonia \\ i.ofodile@ut.ee
}

\author{
Andris Slavinskis \\ Space Technology Department, Tartu Observatory \\ University of Tartu, Estonia \\ andris.slavinskis@ut.ee \\ School of Electrical Engineering \\ Aalto University, Finland
}

\author{
Hendrik Ehrpais \\ Tartu Observatory and Institute of Physics \\ University of Tartu, Estonia \\ hendrik.ehrpais@ut.ee
}

\author{
Gholamreza Anbarjafari \\ iCV Lab, Institute of Technology \\ University of Tartu \\ Tartu, Estonia \\ shb@ut.ee
}

\begin{abstract}
This paper presents the design and study of cross product control, Linear-Quadratic Regulator (LQR) optimal control and high spin rate control algorithms for ESTCube-2/3 missions. The three-unit CubeSat is required to spin up in order to centrifugally deploy a 300-m long tether for a plasma brake deorbiting experiment. The algorithm is designed to spin up the satellite to one rotation per second which is achieved in $\mathbf{4 0}$ orbits. The LQR optimal controller is designed based on closed-loop step response with controllability and stability analysis to meet the pointing requirements of less than $0.1^{\circ}$ for the Earth observation camera and the high-speed communication system. The LQR is based on linearised satellite dynamics with an actuator model. The preliminary simulation results show that the controllers fulfil the requirements set by payloads. While ESTCube-1 used only electromagnetic coils for high spin rate control, ESTCube-2 will make the use of electromagnetic coils, reaction wheels and cold gas thrusters to demonstrate technologies for a deep-space mission ESTCube-3. The attitude control algorithms will be demonstrated in low Earth orbit on ESTCube-2 as a stepping stone for ESTCube-3 which is planned to be launched to lunar orbit where magnetic control is not available.
\end{abstract}

Index Terms-ESTCube-2, attitude control, spin control, control system analysis, feedback linearization, nonlinear systems,

\section{INTRODUCTION}

Several works have aimed to provide solutions to varying attitude determination and control design problems using nanosatellites and microsatellites. These satellites are therefore equipped with reliable attitude control systems for several operational modes and while performing these tasks must maintain stability with disturbance effects on the satellites. ESTCube-2 is the second satellite developed in the Estonian Student Satellite Programme. The programme had previously developed and launched ESTCube- 1 which is a one-unit CubeSat with the main objective to perform the first inorbit electric solar wind sail (E-sail) experiment [1], [2]. The ESTCube-1 Attitude Determination and Control System (ADCS) was able to perform the required satellite spin up for the tether deployment experiment. However, the tether was not deployed due to payload malfunction [3]. ESTCube-1 used three magnetorquers as actuators which produced the magnetic moment of up to $0.1 \mathrm{~A} \cdot \mathrm{m}^{2}$; therefore, relying only on magnetic attitude control. The initially implemented high-rate spin controller [4] had to be redesigned due to the residual magnetic moment which was larger than expected. Modifications were made by developing a coil-correction function that changes the coil output and counter the residual moment [5]. ESTCube-2 is developed to improve upon and expand the previous mission.

ESTCube- 2 is a three-unit CubeSat $\approx 10 \times 10 \times 30 \mathrm{~cm}$ in size and has a mass of $\approx 4 \mathrm{~kg}$. The satellite's main mission is to test the plasma brake which is technology similar to the Esail [6], [7]. The E-sail utilizes charged particles in the solar wind plasma to propel the spacecraft by using conductive tethers via the Coulomb drag. The same can be applied in the Low Earth Orbit's (LEO's) plasma environment to generate a significant amount of Coulomb drag. In addition to the plasma break payload, the satellite is equipped with Earth observation cameras, a high speed communications system, an anti-corrosion experiment, and a science-grade magnetometer. The satellite uses reaction wheels and cold-gas propulsion as well as magnetorquers to complement as actuators and unload reaction wheels. The ADCS of ESTCube-2 is developed for interplanetary environment outside the Earth's magnetosphere which is required for ESTCube-3 to test the E-sail in an authentic solar-wind environment.

The ADCS is required to spin the satellite up to $360 \mathrm{deg} \cdot \mathrm{s}^{-1}$ with an alignment accuracy of less than $3^{\circ}$ which produce the angular momentum for centrifugal deployment of a tether. The ADCS will also fulfill precise satellite pointing of less than $0.1^{\circ}$ in order to operate the Earth observation and high speed communication payloads. It must be able to stabilize and maintain attitude control in the presence of disturbance torques on the satellite. The disturbance torques in LEO includes the magnetic torque, gravity-gradient torque, atmospheric drag and 
solar radiation pressure. The ADCS is designed to tolerate varying disturbance torques within and beyond LEO.

Varying attitude control objectives and problems have been analyzed in several works and literature of specific nanosatellite missions. While earlier works and designs were based on Euler angles spacecraft model, the quaternion model has recently been widely studied [8]-[11]. These works show that the quaternion model has a remarkable advantage over the use of Euler angles. Lyapunov-based functions have been used to design varying attitude control laws [12]-[14]. This, however, may not efficiently globally stabilize a nonlinear system. The use of the quaternion model with linearized dynamics is proven to be controllable and globally stabilizes the nonlinear satellite model [15]. A Proportional-Derivative (PD) like control law also can be used to attain a certain attitude control precision as it is known to asymptotically stabilize the system with application of control torques in three linearly independent directions [16].

The Linear-Quadratic Regulator (LQR) control problem for various applications has been implemented with works relating to CubeSat attitude control [9], [17], [18]. For ESTCube-2, we are studying the LQR feedback gain control methods and presenting results with magnetic attitude control and reaction wheels. We show that the weight matrices are analytically selected based on closed loop step response and that eigenvalues guaranty stability. The LQR optimal control methods presented in this paper can be directly applied to both magnetically and non-magnetically actuated satellites given the actuation model. A process to validate controller gains is also described based on ESTCube-2 parameters and control requirements.

The satellite spin control problem has been studied and implemented on satellites based on a controller approach on spin rate and precession [19]-[21]. A fault-tolerant magnetic spin stabilizing controller was developed for JC2Sat-FF [22]. The implementation of this controller for high spin rate was studied for ESTCube-1, and based on flight results, we redesign the control strategy and its implementation not only to reduce the effect of precession and to minimize nutation but also to ensure a stabilized spin up and obtain optimal control gains to be used in calculations.

This paper is organized as follows. Section II presents the ESTCube-2 Attitude determination and control system design and structure. Section III discusses the satellites mathematical models and linearized models. Section IV describes the design of the attitude control algorithms specific for the ESTCube-2 mission. Section V presents simulation results and analysis of various controller performances. Section VI summarizes the work done on controller design and comparisons.

\section{ESTCube-2 Attitude Determination And CONTROL System Design}

A detailed system design of the ESTCube-2 ADCS is presented in [23]. The ADCS estimates the attitude by fusing measurements of various sensors - star tracker, gyroscopes, magnetometers, Sun sensors and accelerometers. Sun sensors aim to achieve subpixel accuracy based on linear CMOS image sensor that has $1 \times 1024$ pixels. The magnetometers and gyroscopic sensors are based on COTS MEMS sensors. The star tracker, developed in house, is FPGA-based. It is used to obtain a very accurate and precise attitude in combination with other sensors to ensure $0.0125^{\circ}$ accuracy.

ESTCube-2 uses three magnetorquers with a magnetic moment of $0.5 \mathrm{~A} \cdot \mathrm{m}^{-2}$, three reaction wheels for fine attitude control with momentum storage of $1.5 \mathrm{mN} \cdot \mathrm{m} \cdot \mathrm{s}$ and a cold-gas propulsion system with four thrust nozzles in the same $-\mathrm{z}$ axis direction with the nominal thrust of $1 \mathrm{mN}$. Due to the small momentum storage of the reaction wheels, the magnetorquers will be used to prevent saturation by unloading the wheels.

In this study, the satellite is modelled with a mass of $4 \mathrm{~kg}$ and moments of inertia about $\mathrm{x}, \mathrm{y}$ and $\mathrm{z}$ axes $-0.0333 \mathrm{~kg} \cdot \mathrm{m}^{2}$, $0.0333 \mathrm{~kg} \cdot \mathrm{m}^{2}$ and $0.0067 \mathrm{~kg} \cdot \mathrm{m}^{2}$, respectively.

\section{SATEllite Modeling}

Quaternion-based attitude model is used for ESTCube-2 [24, p. 511]. It has several advantages over the Euler rotations: The quaternion attitude representation does not depend on rotation sequence and does not have a singularity point for any attitude. In this preliminary study, we have modeled the following disturbance torques: gravitational, radiation and aerodynamic [25, p. 232-263].

\section{A. Kinematic Equations}

The kinematic equations describing the orientation of the satellite can be represented in quaternions by the differential equations given in [24, Ch. 16].

$$
\begin{gathered}
\dot{q_{v}}=-\frac{1}{2} \omega \times q_{v}+\frac{1}{2} q_{0} \omega \\
\dot{q_{0}}=-\frac{1}{2} \omega^{T} q_{v}
\end{gathered}
$$

where quaternion $q=q_{0}+q_{v}$ consists of Euler symmetric parameters and represents the orientation, and $\omega$ is an angular velocity vector. The kinematics equation of motion using reduced quaternion representation is given in Equation $3[15$, Eq. 57].

$$
\left[\begin{array}{l}
\dot{q_{1}} \\
\dot{q}_{2} \\
\dot{q}_{3}
\end{array}\right]=\frac{1}{2}\left[\begin{array}{ccc}
q_{0} & -q_{3} & q_{2} \\
q_{3} & q_{0} & -q_{1} \\
-q_{2} & q_{1} & q_{0}
\end{array}\right]\left[\begin{array}{l}
\omega_{1} \\
\omega_{2} \\
\omega_{3}
\end{array}\right]
$$

where $q_{1}, q_{2}, q_{3}$ are vector components of $q_{v}$.

\section{B. Dynamic Model}

In modeling the dynamics of the satellite, Newton-Euler formulation is used and describes the angular momentum in relation to applied torques.

$$
J \dot{\omega}_{i}=T_{d}+T_{c}-\Omega\left(\omega_{i}\right) J \omega_{i}
$$

where

- $J$ is inertia matrix

$$
J=\left[\begin{array}{ccc}
J_{x} & -J_{x y} & -J_{x z} \\
-J_{y x} & J_{y} & -J_{y z} \\
-J_{z x} & -J_{z y} & J_{z}
\end{array}\right]
$$


- $J \dot{\omega}_{i}$ is the rate of angular momentum $\dot{h}$ in inertial reference frame

- $\Omega\left(\omega_{i}\right)$ is the skew symmetric representation of the angular velocity in inertial reference frame.

$$
\Omega\left(\omega_{i}\right)=\left[\begin{array}{ccc}
0 & -\omega_{1} & -\omega_{2} \\
\omega_{1} & 0 & \omega_{3} \\
\omega_{2} & -\omega_{3} & 0
\end{array}\right]
$$

- $T_{d}$ is the sum of disturbance torques acting on the satellite

- $T_{c}$ is the applied control input torque.

The varying torques acting on the satellite changes with respect to the selection of actuator for specific attitude operation.

In order to design the controller for precise satellite pointing, the satellite model is described with respect to nadir pointing of the satellite with reaction wheels.

$$
J \dot{\omega}_{i}=T_{d}+T_{c}-\Omega\left(\omega_{i}\right)\left(J \omega_{i}+H\right)
$$

where $H=\left[\begin{array}{lll}h_{1} & h_{2} & h_{3}\end{array}\right]^{T}$ is the angular momentum of the wheel. For this, the attitude is represented by body frame rotation relative to the orbit frame (LVLH). By application of the transformation matrix $A_{o}^{B}$, the angular velocity of the satellite in inertial reference frame is given as

$$
\omega_{i}=\omega+A_{o}^{B} \omega_{o}=\omega+\omega_{o}^{B}
$$

where $\omega$ is described as the angular velocity in the body frame with respect to the orbit frame. Based on the derivative of $\omega_{i}$, and assuming that $\dot{\omega}_{o}$ is small and negligible, Equation 7 can be re-written as

$$
J \dot{\omega}_{i}=T_{d}+T_{c}-\Omega\left(\omega+\omega_{o}^{B}\right)\left(J\left(\omega+\omega_{o}^{B}\right)+H\right)
$$

\section{Linearized Satellite Model}

In order to design the LQR optimal controller, the non-linear satellite equations have to be linearized. The linearization of the satellite attitude equations can be obtained by linearizing the equations about an equilibrium or stationary point. The attitude equilibrium point is set as $q_{0}=1$ and $q_{1}, q_{2}, q_{3}=0$ for the linearization based on a first order Taylor expansion [15, Sec. 3.2.2]. For this, Equation 3 can be represented as a function $g$ of $q$ and $w_{i}$ and its partial derivative given as

$$
\dot{q_{v}}=\frac{\partial g}{\partial q}(q)+\frac{\partial g}{\partial \omega_{i}}\left(\omega_{i}\right)
$$

with a change around equilibrium point eq, where at equilibrium $\omega_{i}=0$.

$$
\dot{q}-q_{e q}=\frac{\partial g}{\partial q}\left(q-q_{e q}\right)+\frac{\partial g}{\partial \omega_{i}}\left(\omega_{i}-\omega_{i e q}\right)
$$

With respect to the above expression, Equation 3 is therefore expressed as

$$
\left[\begin{array}{c}
\dot{q_{1}} \\
\dot{q_{2}} \\
\dot{q_{3}}
\end{array}\right]=\left[\begin{array}{cccccc}
0 & 0 & 0 & \frac{1}{2}\left(q_{0}\right) & 0 & 0 \\
0 & 0 & 0 & 0 & \frac{1}{2}\left(q_{0}\right) & 0 \\
0 & 0 & 0 & 0 & 0 & \frac{1}{2}\left(q_{0}\right)
\end{array}\right]\left[\begin{array}{c}
q_{1} \\
q_{2} \\
q_{3} \\
\omega_{1} \\
\omega_{2} \\
\omega_{3}
\end{array}\right]
$$

Therefore with approximation of Equation $4\left(J \dot{\omega}_{i} \approx T_{c}\right)$ with negligible disturbance torque and $q_{0}=1$ at equilibrium, the state space model where $u=T_{c}$ can be obtained.

$$
\dot{x}=A x+B u
$$

$$
A=\left[\begin{array}{cc}
0_{3} & \frac{1}{2}\left(I_{3}\right) \\
0_{3} & 0_{3}
\end{array}\right], x=\left[\begin{array}{c}
q_{v} \\
\omega_{i}
\end{array}\right], B=\left[\begin{array}{c}
0_{3} \\
J^{-1}
\end{array}\right]
$$

The linearized model for nadir pointing of ESTCube-2 with reaction wheels can be obtained from Equation 9 with the angular momentum of the wheel small and neglected in the approximation and for simplicity written as $J \dot{\omega}_{i}=T_{d}+T_{c}+$ $f\left(\omega, \omega_{o}^{B}\right)$. With the orbit rate of the satellite $\omega_{o}=2 \pi / T_{o}$, the model in state space form with gravity gradient disturbance torque is given as described in [15].

$$
\begin{gathered}
{\left[\begin{array}{c}
\dot{q}_{v} \\
\dot{\omega}_{i}
\end{array}\right]=\left[\begin{array}{cccccc}
0 & 0 & 0 & 0.5 & 0 & 0 \\
0 & 0 & 0 & 0 & 0.5 & 0 \\
0 & 0 & 0 & 0 & 0 & 0.5 \\
f_{41} & 0 & 0 & 0 & 0 & f_{46} \\
0 & f_{52} & 0 & 0 & 0 & 0 \\
0 & 0 & f_{63} & f_{64} & 0 & 0
\end{array}\right]\left[\begin{array}{c}
q_{v} \\
\omega_{i}
\end{array}\right]+} \\
\\
{\left[\begin{array}{ccc}
0 & 0 & 0 \\
0 & 0 & 0 \\
0 & 0 & 0 \\
1 & 0 & 0 \\
0 & 1 & 0 \\
0 & 0 & 1
\end{array}\right]\left(T_{c}\right)}
\end{gathered}
$$

where

$$
\begin{array}{r}
f_{41}=8\left(J_{z}-J_{y}\right) \omega_{o}^{2}-2 \omega_{o} \\
f_{46}=\left(-J_{x}-J_{z}+J_{y}\right) \omega_{o} \\
f_{52}=6\left(J_{z}-J_{x}\right) \omega_{o}^{2} \\
f_{63}=2\left(J_{x}--J_{y}\right) \omega_{o}^{2}-2 \omega_{o} \\
f_{64}=-f_{46}
\end{array}
$$

The linearized model with magnetorquers is similar, however the control torque used is generated by the magnetic coil as

$$
T_{c}=m \times b
$$

where $m$ is the magnetic dipole moment from the electromagnetic coils, and $b$ is an approximation of the magnetic field [24, Ap. $\mathrm{H}]$ and is expressed based on the magnetic dipole moment as

$$
b=\left[\begin{array}{l}
b_{1}(t) \\
b_{2}(t) \\
b_{3}(t)
\end{array}\right]=\frac{\mu_{f}}{a^{3}}\left[\begin{array}{c}
\cos \omega_{0} t \sin \theta \\
-\cos \theta \\
2 \sin \omega_{0} t \sin \theta
\end{array}\right]
$$


By assuming and approximating the inclination of the satellite orbit and magnetic equator $\theta=0$, the quaternion linear time-invariant model is reduced and given by [26].

$$
\begin{gathered}
{\left[\begin{array}{c}
\dot{q}_{v} \\
\dot{\omega}_{i}
\end{array}\right]=\left[\begin{array}{cccccc}
0 & 0 & 0 & 0.5 & 0 & 0 \\
0 & 0 & 0 & 0 & 0.5 & 0 \\
0 & 0 & 0 & 0 & 0 & 0.5 \\
f_{41} & 0 & 0 & 0 & 0 & f_{46} \\
0 & f_{52} & 0 & 0 & 0 & 0 \\
0 & 0 & f_{63} & f_{64} & 0 & 0
\end{array}\right]\left[\begin{array}{c}
q_{v} \\
\omega_{i}
\end{array}\right]} \\
+\left[\begin{array}{cccc}
0 & 0 & 0 \\
0 & 0 & 0 \\
0 & 0 & 0 \\
0 & 0 & -b_{2} / J_{x} \\
0 & 0 & 0 \\
-b_{2} / J_{z} & 0 & 0
\end{array}\right]\left[\begin{array}{c}
m_{1} \\
m_{2} \\
m_{3}
\end{array}\right]
\end{gathered}
$$

where

$$
\begin{array}{r}
f_{41}=8 \frac{\left(J_{z}-J_{y}\right)}{J_{x}} \omega_{o}^{2} \\
f_{46}=\frac{\left(-J_{x}-J_{z}+J_{y}\right)}{J_{x}} \omega_{o} \\
f_{52}=6 \frac{\left(J_{z}-J_{x}\right)}{J_{y}} \omega_{o}^{2} \\
f_{63}=2 \frac{\left(J_{x}-J_{y}\right)}{J_{z}} \omega_{o}^{2} \\
f_{64}=\frac{\left(J_{x}+J_{z}-J_{y}\right)}{J_{z}} \omega_{o}
\end{array}
$$

\section{Attitude Control Algorithms}

The design of attitude control algorithms for ESTCube-2 is based on the quaternion model of the satellite and its linearized models. The desired satellite quaternion for the control algorithms are expressed as error quaternions with measured quaternion values as in Equation 22

$$
q_{e}=\left[\begin{array}{cccc}
q_{d 0} & q_{d 1} & -q_{d 2} & -q_{d 3} \\
-q_{d 1} & q_{d 0} & q_{d 3} & -q_{d 2} \\
q_{d 2} & -q_{d 3} & q_{d 0} & -q_{d 1} \\
q_{d 3} & q_{d 2} & q_{d 1} & q_{d 0}
\end{array}\right]\left[\begin{array}{c}
q_{0} \\
q_{1} \\
q_{2} \\
q_{3}
\end{array}\right]
$$

The quaternion error $q_{e}$ satisfies the unit quaternion constraint. Where $q_{d}$ is the desired satellite attitude.

\section{A. B-dot Controller}

The B-dot is derived by observing a decrease in the rotational energy during detumbling. This means that the scalar product of the angular velocity and the control torque must be negative

$$
\omega_{i}^{T} \cdot \tau<0
$$

where $\tau$ is represented as the control torque delivered by magnetic actuator and is expressed as

$$
\tau=m \times B
$$

where $m$ is the commanded magnetic dipole moment and $B$ is the geomagnetic field vector. A seemingly accurate model of the geomagnetic field for LEO circular orbit such

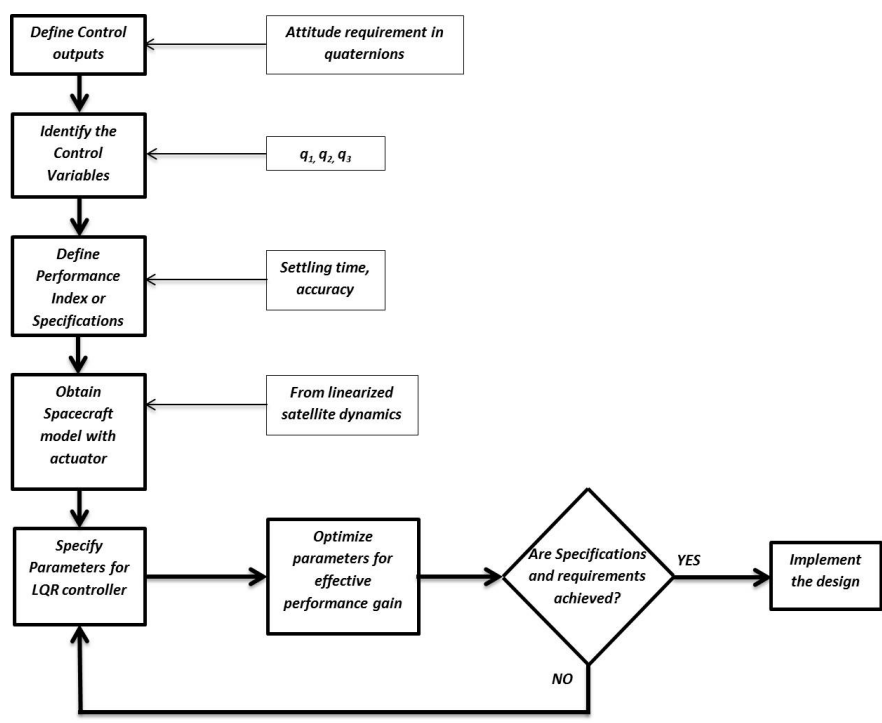

Fig. 1: LQR controller design algorithm.

as International Geomagnetic Reference Field (IGRF) is being used as the model for the simulation.

In order to decrease the kinetic energy of the spacecraft the control torque $\tau$ has to be proportional to $-\omega$ and based on the above inequality, the magnetic moment needs to be perpendicular to $\omega \times B$ as no torque will be produced if it were parallel. We can therefore complete the solution by implementing a scalar gain $k$

$$
m=-k \cdot\left(\omega_{i} \times B\right)
$$

where $k$ is a positive gain. The change in magnetic field vector is assumed to be mainly as a result of rotation of the satellite

$$
\dot{B} \approx\left(\omega_{i} \times B\right)
$$

therefore we can obtain a simple control law based on

$$
m=-k \dot{B}
$$

\section{B. Proportional-Derivative (PD)}

The PD controller designed for the model is implemented for both magnetic attitude control and with reaction wheels. The control torque vector derived is given as

$$
T_{c}=\left[k_{\omega} \omega_{i}+k_{q} q_{e}\right]
$$

The PD magnetic control law is defined with respect to the Earth's magnetic field $B$

$$
m=k_{\omega}\left(\omega_{i} \times B\right)+k_{q}\left(q_{e} \times B\right)
$$

where $m$ is the commanded magnetic moment of the magnetorquers. 


\section{Cross Product Law}

Due to the limitation of the angular momentum of the reaction wheel used on ESTCube- 2 at $1.5 \mathrm{mN} \cdot \mathrm{m} \cdot \mathrm{s}$, the full controllability of the satellite for attitude maneuvers requires that the wheels are desaturated. Several approaches for wheel desaturation and unloading have been discussed to solve this [27]-[29]. Here a simple cross product law based on the PD control is implemented to perform the pointing of the satellite by constantly verifying the angular momentum of the wheel as a feedback and enabling the magnetorquer in the algorithm when the wheel gets saturated.

$$
m=-\frac{k}{(\|B\|)^{2}}\left[B \times h_{e}\right]
$$

$m$ is the magnetorquer dipole moment vector in SBRF and $h_{e}$ is the angular momentum error of the wheels.

\section{Linear-Quadratic Regulator $(L Q R)$}

The LQR control technique is designed mainly for attitude stabilization during pointing. The design will be implemented based on the linearized satellite model in Section III-C. The design aims to find a cost function and minimize this cost function. The design based on state space quaternion approach begins with a basic feedback control of $u=-K x$ expressed further in Equation 31 where $K$ is the gain matrix obtained to minimize the linear-quadratic cost Function 32

$$
\begin{gathered}
u=-R^{-1} B^{T} P x \\
J=\int_{0}^{\infty}\left[x^{T} Q x+u^{T} R u\right] d t
\end{gathered}
$$

where $x$ is a vector of system states, $A$ is the system state matrix, $B$ is the input matrix, $Q$ and $R$ are known as the state weight matrix and control input weight matrix respectively and $P$ is a symmetric positive semi-definite solution of the algebraic Riccati equation given below

$$
0=P A+A^{T} P+Q-P B R^{-1} B P
$$

The algebraic Riccati equation is only solvable if the input matrices $\mathrm{A}$ and $\mathrm{B}$ are controllable. The controllability of $\mathrm{A}$ and $B$ aims to satisfy the controllability property of

$$
C_{o}=\left[B|A B| A^{2} B|\cdots| A^{n-1} B\right]
$$

where $n$ is the dimension of $A$ and the system is therefore controllable if and only if $C_{o}$ has a full $\operatorname{rank}, \operatorname{rank}\left(C_{o}\right)=n$. The controllability of the designed system is determined by using MATLAB controllability function ctrb. Figure 1 shows the design process for the LQR optimal control.

By adjusting the values of the $Q$ and $R$ matrices, a comparison can be made to the step response performance to attain the desired result. Using MATLAB, the feedback gain $K$ can be obtained using the $l q r$ function given as: $[K, P, E]=\operatorname{lqr}(A, B, Q, R) . P$ is obtained as a solution to the algebraic Riccati equation given in Equation 33 and $E$ is the closed loop eigen values $|A-B K|$ which must guarantee stability. The values of $Q$ and $R$ can be investigated by applying a step input for steady state response of the system to the angular velocity and by evaluating responses, an optimal controller gain can be derived. Figure 2 shows the step response to the angular velocity with $Q$ and $R$ matrices as $I_{6}$ and $I_{3}$ respectively.

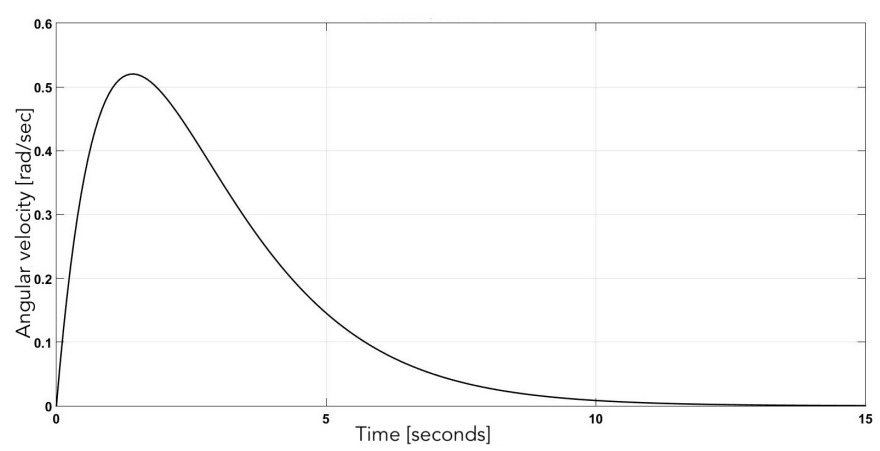

Fig. 2: Steady-state closed-loop LQR step response.

\section{E. Angular Rate Spin Control}

The spin up control algorithm fulfills the mission requirement of spinning the satellite to achieve an angular velocity of $360 \mathrm{deg} \cdot \mathrm{s}^{-1}$ to provide angular momentum for the centrifugal tether deployment for the plasma break experiment. The spin motion control of the satellite is based on three important control factors that should be considered: spin control, precession control and nutation control [30]. For this preliminary study, the controller uses only magnetorquers. The control algorithm in Equations 35 and 36 is designed for spin control and is based on a Lyapunov stability function which reduces the effect of precession and minimises nutation [22].

$$
\begin{gathered}
A=B \times\left(\tilde{h}+k_{1} \tilde{h}_{x}\left[\begin{array}{l}
1 \\
0 \\
0
\end{array}\right]+k_{2} P \omega\right) \\
m=\operatorname{sat}\left[-\frac{k}{(\|B\|)^{2}} A\right]
\end{gathered}
$$

where $B$ is the Earth's magnetic field vector in Satellite Body Reference Frame (SBRF), $\tilde{h}$ is the satellite angular momentum error in SBRF, $\tilde{h}_{x}$ is the angular momentum error around the satellite's $\mathrm{x}$ axis, $P$ is the selection matrix for the $\mathrm{y}$ and $\mathrm{z}$ axes, $\omega$ is the angular velocity vector in SBRF, $m$ is the magnetorquer dipole moment vector in SBRF and $k, k_{1}, k_{2}$ are control law gains. More details of a controller for a similar mission, ESTCube-1, are provided in [4].

In obtaining preliminary results for the optimal performance of the controller, the following points are noted:

- The B-dot algorithm described in Section IV-A for detumbling of the satellite should first be used to reduce the angular momentum of the satellite in order to improve the performance of the spin up algorithm.

- For the iterative loop in running the controller, the initial desired angular velocity should be approximately $45 \mathrm{deg} \cdot \mathrm{s}^{-1}$ to ensure stability along the spin axis. 
- The value of the control gains should be set as: $k_{2}>1, k_{1}<1, k>>k_{1}$ to augment the nutation damping process and avoid uncontrolled spinning of the satellite about the transverse axis.

\section{Simulation Results and Discussions}

The attitude control algorithms are designed and tested with their performance evaluated using a custom-built attitude simulator in MATLAB and Simulink. The simulation environment includes the following features:

- Basic spacecraft dynamics based on the Euler equations;

- Environmental disturbances;

- Realistic actuator and sensor models.

\section{A. B-dot}

Figure 3 shows the result of the simulation of the B-dot controller with a controller gain of $12 \times 10^{4}$. Initial inspection of the controller shows that it performs as expected but becomes very slow on converging at steady state. The response shown is based on setting the frequency to $100 \mathrm{~Hz}$. The initial angular rate was set at $17 \mathrm{deg} \cdot \mathrm{s}^{-1}$ in the $\mathrm{z}$-axis.

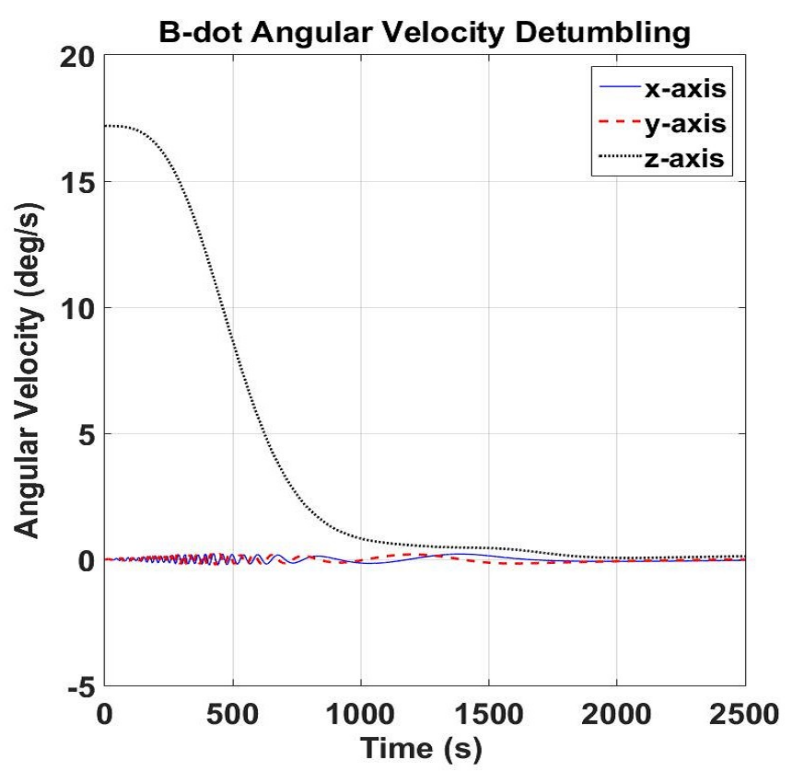

Fig. 3: Detubmling with B-dot controller.

\section{B. Cross Product law}

The cross product control law is implemented with torque commands from both reaction wheels and magnetorquers. The initial angular velocity of the satellite was set at $13.5 \mathrm{deg} \cdot \mathrm{s}^{-1}$ in the x-axis. As seen in Figure 4, the angular velocity of the satellite attenuates to $0^{\circ}$ in about $1000 \mathrm{~s}$. The attitude of the satellite attempts to attain nadir pointing stabilization mode in about $4000 \mathrm{~s}$. The reaction wheels angular momentum saturates to $1.5 \mathrm{mN} \cdot \mathrm{m} \cdot \mathrm{s}$ in about $100 \mathrm{~s}$, as seen in Figure 4 . The magnetorquers are then activated and delivers torque which also desaturates the wheels and regulates performance for the satellite pointing mode.
TABLE I: Spin rate simulation parameters.

\begin{tabular}{|c|c|}
\hline Parameter & Value \\
\hline Initial Angular Velocity & {$\left[\begin{array}{lll}0 & 0 & 0\end{array}\right]^{T} \mathrm{deg} \cdot \mathrm{s}^{-1}$} \\
\hline $\mathrm{k}$ & 15000 \\
\hline$k_{1}$ & 0.01 \\
\hline \hline Desired Angular Velocity, deg $\cdot \mathrm{s}^{-1}$ & $k_{2}$ Gain \\
\hline 40 & 100 \\
\hline 97 & 1000 \\
\hline 155 & 1000 \\
\hline 195 & 1000 \\
\hline 252 & 5000 \\
\hline 298 & 5000 \\
\hline 360 & 5000 \\
\hline
\end{tabular}

\section{Linear-Quadratic Regulator}

The LQR optimal control was designed and simulated for attitude control with magnetorquers and reaction wheels. The initial values for the $Q$ and $R$ matrices were selected by finding the optimal step response performance of the controller with varying weight matrices. For further simplification of the design process, the weight matrices $Q$ and $R$ are selected to be diagonal with the number of states and number of actuator control as the lengths $Q$ and $R$ respectively.

$$
\begin{array}{r}
Q=I_{6}\left[Q_{1}, Q_{2}, Q_{3}, Q_{4}, Q_{5}, Q_{6}\right] \\
R=I_{3}\left[R_{1}, R_{2}, R_{3}\right]
\end{array}
$$

Figure 5 shows the LQR controller performance with reaction wheel, when the $Q$ and $R$ matrices were selected as $Q=I_{6}[1,1,1,1,1,1]$ and $R=I_{3}[1,1,1]$. The result of the closed loop eigenvalues were observed to ensure stability and the controller gain $K$ obtained. The result shows that the controller achieves about $0 \mathrm{deg} \cdot \mathrm{s}^{-1}$ angular velocity settling time in $100 \mathrm{~s}$ on all axes. Further results show the controller performs disturbance rejection with varying disturbances.

Figures 6 shows results of the LQR controller with magnetorquers. The angular velocity of the satellite is seen to attain rest at $0 \mathrm{deg} \cdot \mathrm{s}^{-1}$ in all axes in about $1500 \mathrm{~s}$. The controller gain was calculated with $\mathrm{Q}$ and $\mathrm{R}$ matrices selected as $Q=I_{6}[140,140,140,140,140,140]$ and $R=$ $I_{3}[2480,2480,2480]$.

\section{Angular Rate Spin Control}

The spin control result is presented in Figure 7 with the desired ultimate angular rate set to $360 \mathrm{deg} \cdot \mathrm{s}^{-1}$ in the $\mathrm{X}$-axis. As described in Section IV-E, the controller gains were set as shown in Table I. The result shows the desired angular rate which is performed in several phases is achieved in about 40 orbits as enumerated in Table I. The angular rate then stabilizes at $360 \mathrm{deg} \cdot \mathrm{s}^{-1}$ for a few more orbits. 

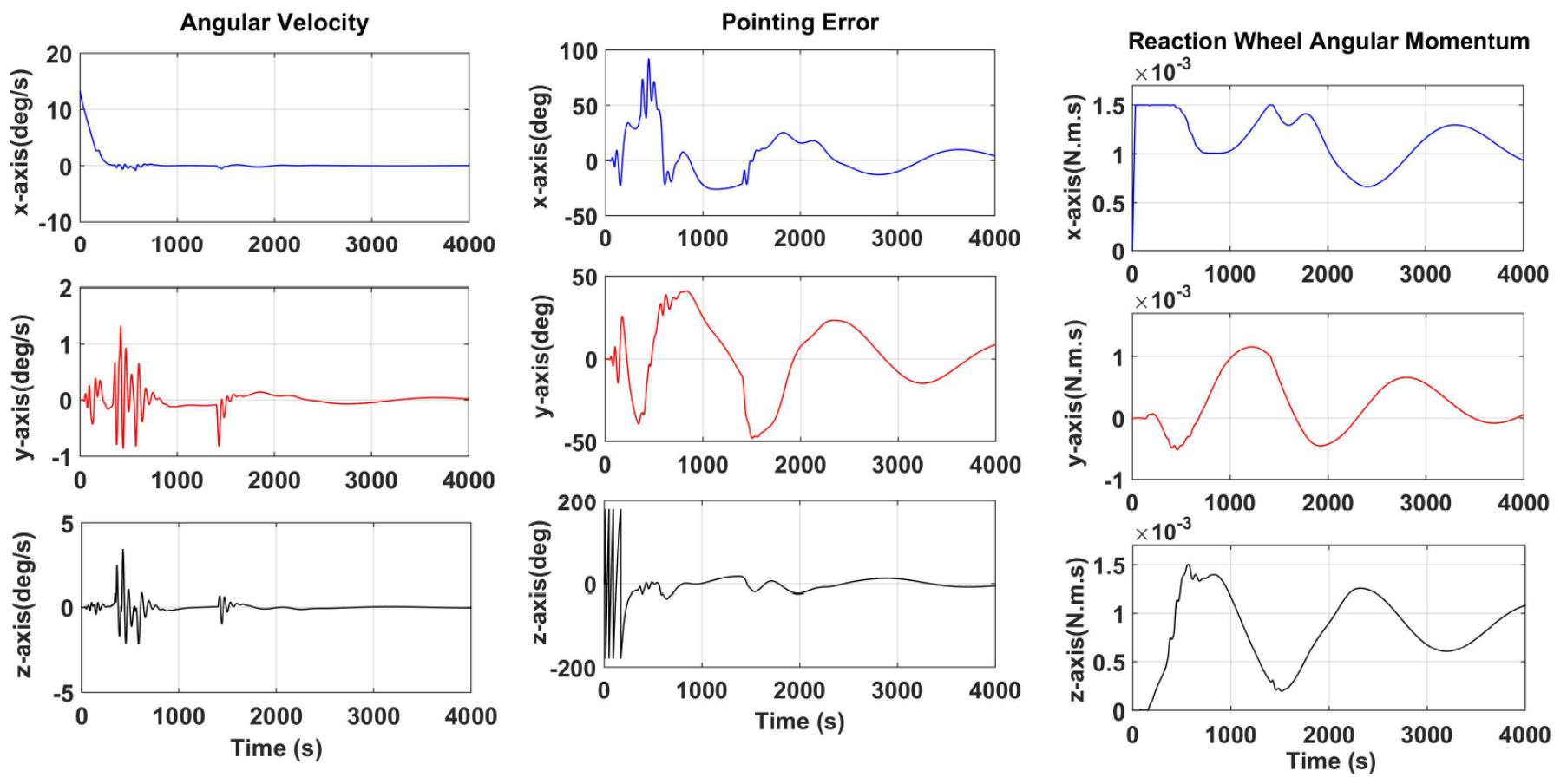

Fig. 4: Unloading reaction wheels with cross product control law and magnetorquers.
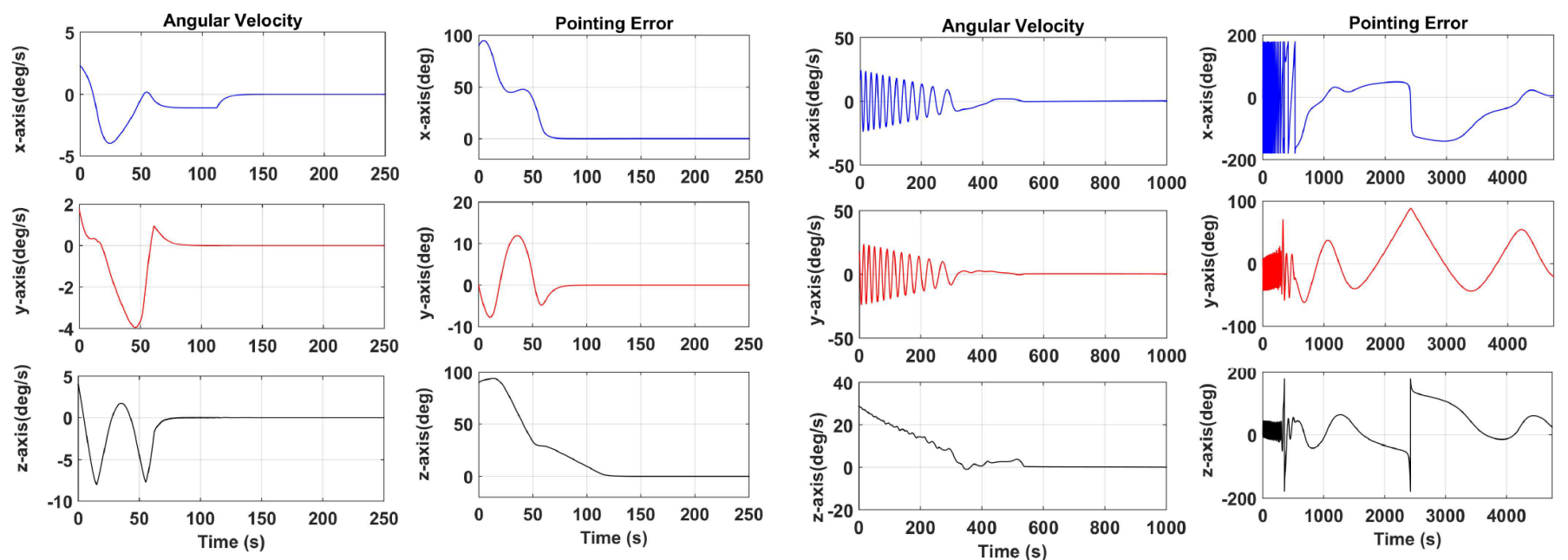

Fig. 5: Attitude control with LQR and reaction wheels.

\section{CONCLUSIONS AND FUTURE WORK}

The result of the study reflected various preliminary performance characteristics for the controllers for attitude maneuvers. The cross product law efficiently performed the unloading of saturated reaction wheels in order to propagate precise satellite pointing and attain stability. The angular rate spin control needs to be implemented as indicated in the setup as the spin up of the $\mathrm{x}$-axis is known to disturb the transverse axis if the requirements of the spin rate are not adhered to. The design of LQR optimal controller for use with the reaction wheels and magnetorquers was shown to work well even with the gravity-gradient disturbance torque.
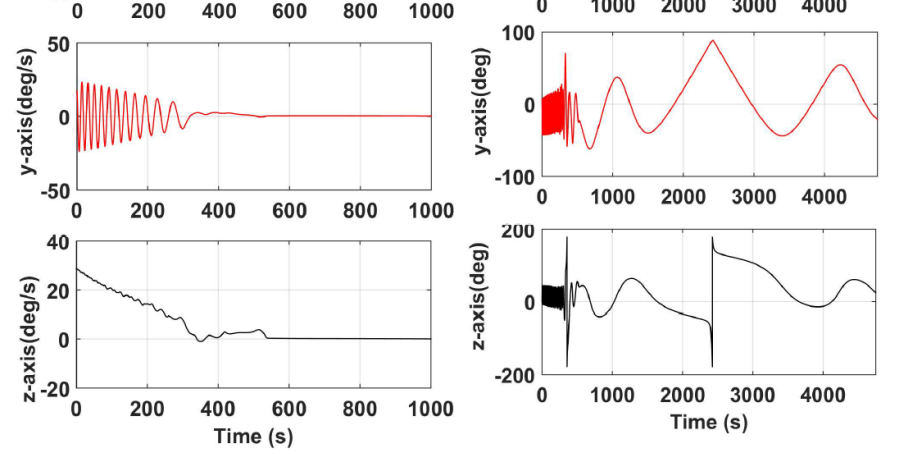

Fig. 6: Attitude control with LQR and magnetorquers.

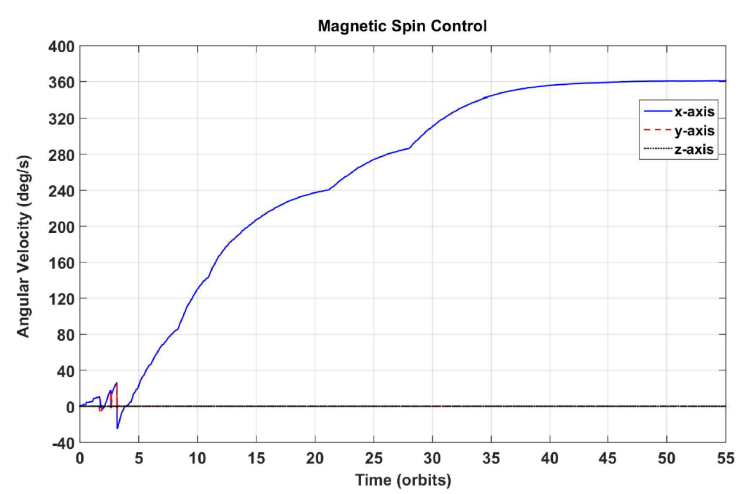

Fig. 7: Angular rate spin up control performance. 
However, the response with magnetorquers is less desirable in comparison with reaction wheels. In comparison with the PD-like control laws, LQR optimal control gave better results to stabilize the system even when realistic disturbances were added.

To attain a more efficient performance, controllers making use of the magnetorquers as actuators would be optimized in terms controller gain and testing of time varying gain approach for the final satellite design.

In the future, a more realistic model of the satellite will be used and disturbance analysis will be presented. This includes a more accurate inertia tensor, the misalignment between torquers, satellite body frame and the principal inertia axes. In addition, detailed analysis will be provided regarding the placement and the properties of the torquers, how that affects attitude control, as well as additional details on spin control laws and their performance with reaction wheels and thrusters. The saturation of reaction wheels during control maneuvers and stabilisation due to disturbance torques will be analysed. We will also consider other approaches for linearisation: Instead of analytically linearising the equations around one specific target, the system could be numerically linearised at each time step. Such an approach might provide a more optimal way to control the satellite. Alternatively, non-linear control or the linearised nadir-pointing spacecraft model can be used [15, Sec. 3.3.2].

\section{ACKNOWLEDGMENT}

The authors would like to thank everybody who has contributed to the development of ESTCube-2 and its attitude determination and control system. We would also like to thank all the partners involved in developing the ESTCube-2 nanosatellite. The research for this article was partly supported by the University of Tartu ASTRA project 2014-2020.4.01.160029 KOMEET "Benefits for Estonian Society from Space Research and Application", financed by the EU European Regional Development Fund, Estonian Research Council Grants (PUT638), the Scientific and Technological Research Council of Turkey (TÜBITAK) (116E097), and the Estonian Centre of Excellence in IT (EXCITE) funded by the European Regional Development Fund. We thank three anonymous reviewers for a thorough and constructive feedback which helped to improve the paper.

\section{REFERENCES}

[1] P. Janhunen, "Electric sail for spacecraft propulsion," J. Propul. Power, vol. 20, pp. 763-764, 2004.

[2] S. Lätt, A. Slavinskis, E. Ilbis, U. Kvell, K. Voormansik, E. Kulu, M. Pajusalu, H. Kuuste, I. Sünter, T. Eenmäe et al., "Estcube-1 nanosatellite for electric solar wind sail in-orbit technology demonstration," Proceedings of the Estonian Academy of Sciences, vol. 63, no. 2, p. 200, 2014.

[3] A. Slavinskis, M. Pajusalu, H. Kuuste, E. Ilbis, T. Eenmäe, I. Sünter, K. Laizans, H. Ehrpais, P. Liias, E. Kulu et al., "ESTCube-1 in-orbit experience and lessons learned," IEEE Aerospace and Electronic Systems Magazine, vol. 30, no. 8, pp. 12-22, 2015.

[4] A. Slavinskis, U. Kvell, E. Kulu, I. Sünter, H. Kuuste, S. Lätt, K. Voormansik, and M. Noorma, "High spin rate magnetic controller for nanosatellites," Acta Astronaut., vol. 95, pp. 218-226, 2014.
[5] H. Ehrpais, J. Kütt, I. Sünter, E. Kulu, A. Slavinskis, and M. Noorma, "Nanosatellite spin-up using magnetic actuators: ESTCube-1 flight results," Acta Astronaut., pp. 210-216, 2016.

[6] I. Iakubivskyi, H. Ehrpais, H. Kuuste, I. Sünter, E. Ilbis, M.-L. Aru, E. Oro, J. Kütta, P. Toivanen, P. Janhunen, and A. Slavinskis, "Estcube2 plasma brake payload for effective deorbiting," 2017.

[7] P. Janhunen, "Simulation study of the plasma-brake effect," Annales Geophysicae, vol. 32, no. 10, pp. 1207-1216, 2014.

[8] B. W. Young, "Design and specification of an attitude control system for the dande mission," Master's thesis, University of Colorado at Boulder, 2008.

[9] N. Jovanovic, "Aalto-2 satellite attitude control system," 2014.

[10] M. Blanke and M. B. Larsen, "Satellite dynamics and control in a quaternion formulation," Technical University of Denmark, Department of Electrical Engineering, Tech. Rep., 2010.

[11] C. Kaplan, "Leo satellites: attitude determination and control components; some linear attitude control techniques," Master's thesis, MIDDLE EAST TECHNICAL UNIVERSITY, 2006.

[12] J.-Y. Wen and K. Kreutz-Delgado, "The attitude control problem," IEEE Transactions on Automatic control, vol. 36, no. 10, pp. 1148-1162, 1991.

[13] J. D. Boškovic, S.-M. Li, and R. K. Mehra, "Robust adaptive variable structure control of spacecraft under control input saturation," Journal of Guidance, Control, and Dynamics, vol. 24, no. 1, pp. 14-22, 2001.

[14] R. J. Wallsgrove and M. R. Akella, "Globally stabilizing saturated attitude control in the presence of bounded unknown disturbances," Journal of Guidance, Control, and Dynamics, vol. 28, no. 5, pp. 957963, 2005

[15] Y. Yang, "Spacecraft attitude determination and control: Quaternion based method," Annual Reviews in Control, vol. 36, no. 2, pp. 198 219,2012

[16] B. Baghi, M. Kabganian, R. Nadafi, and E. Arabi, "Three-axis attitude stabilization of a flexible satellite using non-linear pd controller," Transactions of the Institute of Measurement and Control, vol. 40, no. 2, pp. 591-605, 2018.

[17] F. G. Stray, "Attitude control of a nano satellite," Master's thesis, 2010.

[18] G. Bråthen, "Design of attitude control system of a double cubesat," Master's thesis, Institutt for teknisk kybernetikk, 2013.

[19] R. Pfaff, C. Carlson, J. Watzin, D. Everett, and T. Gruner, "An overview of the fast auroral snapshot (fast) satellite," in The FAST Mission. Springer, 2001, pp. 1-32.

[20] K. Vega, D. Auslander, and D. Pankow, "Design and modeling of an active attitude control system for cubesat class satellites," in AIAA Modeling and Simulation Technologies Conference, 2009, p. 5812.

[21] M. Shigehara, "Geomagnetic attitude control of an axisymmetric spinning satellite," Journal of spacecraft and rockets, vol. 9, no. 6, pp. 391$398,1972$.

[22] A. de Ruiter, "A fault-tolerant magnetic spin stabilizing controller for the JC2Sat-FF mission," Acta Astronaut., vol. 68, pp. 160-171, 2011.

[23] I. Ofodile, J. Kütt, M. K. Nigol, J. Kivastik, A. Parelo, E. Ilbis, H. Ehrpais, and A. Slavinskis, "ESTCube-2 attitude determination and control: Step towards interplanetary CubeSats," in IEEE Aerospace Conference, 2019

[24] J. R. Wertz, Ed., Spacecraft Attitude Determination and Control. Kluwer Academic Publishers, 1978.

[25] P. C. Hughes, Spacecraft Attitude Dynamics. Dover Publications, 2004.

[26] Y. Yang, "Controllability of spacecraft using only magnetic torques," IEEE Transactions on Aerospace and Electronic Systems, vol. 52, no. 2, pp. 954-961, 2016.

[27] J.-F. Trégouët, D. Arzelier, D. Peaucelle, C. Pittet, and L. Zaccarian, "Reaction wheels desaturation using magnetorquers and static input allocation," IEEE Transactions on Control Systems Technology, vol. 23 , no. 2, pp. 525-539, 2015.

[28] X. Chen, W. H. Steyn, S. Hodgart, and Y. Hashida, "Optimal combined reaction-wheel momentum management for earth-pointing satellites," Journal of guidance, control, and dynamics, vol. 22, no. 4, pp. 543$550,1999$.

[29] M. J. Sidi, Spacecraft dynamics and control: a practical engineering approach. Cambridge university press, 1997, vol. 7.

[30] R. Reynolds and G. Creamer, "Global lyapunov control of spin stabilized spacecraft," in 2001 Flight Mechanics Symposium, vol. 1, 2001. 